\title{
Immunoglobulin G4 Sclerosing Cholangitis: An Unusual Cause of Obstructive Jaundice-Case Report and Literature Review
}

\author{
Pragya Shrestha $\mathbb{D}^{1},{ }^{1}$ Brian Le $\mathbb{D}^{2},{ }^{2}$ Brent Wagner, ${ }^{3}$ William Pompella, ${ }^{1}$ \\ and Paras Karmacharya $\mathbb{D}^{1,4}$ \\ ${ }^{1}$ Department of Medicine, Reading Hospital-Tower Health System, West Reading, PA 19611, USA \\ ${ }^{2}$ Department of Pathology, Reading Hospital-Tower Health System, West Reading, PA 19611, USA \\ ${ }^{3}$ Department of Radiology, Reading Hospital-Tower Health System, West Reading, PA 19611, USA \\ ${ }^{4}$ Department of Rheumatology, Mayo Clinic, Rochester, MN 55905, USA
}

Correspondence should be addressed to Pragya Shrestha; sh.pragya@gmail.com

Received 25 May 2018; Accepted 19 July 2018; Published 23 August 2018

Academic Editor: Tsai-Ching Hsu

Copyright (c) 2018 Pragya Shrestha et al. This is an open access article distributed under the Creative Commons Attribution License, which permits unrestricted use, distribution, and reproduction in any medium, provided the original work is properly cited.

\begin{abstract}
IgG4-related sclerosing cholangitis (IgG4-SC) is one of the most common extra-pancreatic manifestation of IgG4-related disease (IgG4-RD) and is clinically distinct from primary sclerosing cholangitis (PSC). IgG4-RD is an increasingly recognized immunemediated fibroinflammatory systemic disease, mostly affecting middle-aged and older male populations that can affect multiple organs. The presence of extra-biliary clinical manifestations of IgG4-RD, such as parotid and lacrimal swelling, lymphadenopathy, autoimmune pancreatitis, and retroperitoneal fibrosis, if present could provide important clues to diagnosis. High serum IgG4 levels, characteristic radiological (e.g., sausage-shaped pancreas or periaortitis) or biopsy findings (high percentage of IgG4+ plasma cells, lymphoplasmacytic infiltrate, storiform fibrosis, or obliterative phlebitis) in the setting of these features is diagnostic of this disease process. However, isolated IgG4-SC might be a diagnostic challenge, and the distinction is important as management of this disorder is vastly different from other causes of cholangitis such as PSC. Systemic corticosteroid therapy is the mainstay of therapy.
\end{abstract}

\section{Introduction}

Immunoglobulin G4-related sclerosing cholangitis (IgG4SC) is a relatively uncommon but increasingly recognized entity. It was first described in 2001 by Hamano et al. [1] with a landmark study demonstrating elevated levels of serum IgG4 in patients with sclerosing cholangitis [1-4]. It is characterized by systemic inflammatory and sclerosing lesions with massive infiltration of IgG4-positive lymphocytes involving multiple organ systems, such as the eyes, salivary glands, lacrimal glands, lungs, pancreas, kidneys, retroperitoneum, and vascular system $[2,5]$. Sclerosing cholangitis (SC) is one of the common organ manifestations of IgG4-related disease (IgG4-RD), affecting approximately $60 \%$ of patients with this systemic disease [5]. It may occur as a part of the systemic manifestation of IgG4-RD, often associated with type 1 autoimmune pancreatitis $[1,2,6]$.
Isolated IgG4-SC can occur rarely and pose a diagnostic challenge $[5,6]$.

\section{Case Presentation}

An 81-year-old male presented to the clinic with yellowish discoloration of skin and urine for 2 weeks. He denied any fever, abdominal pain, nausea, vomiting, melena, hematochezia, or acholic stools. Past medical history was significant for hypertension, hyperlipidemia, diabetes mellitus type II, coronary artery disease, and chronic kidney disease stage IV. He reported recent loss of appetite but denied any significant weight changes. Ultrasound ordered by primary care physician showed intra- and extra-hepatic biliary dilation with distension of gall bladder without cholelithiasis. He was sent to the emergency department (ED) for further evaluation. 
On examination, blood pressure was $133 / 60 \mathrm{mmHg}$, heart rate was 75 beats per minute, respiratory rate was 23 breaths per minute, temperature was $97.7^{\circ} \mathrm{F}$, and oxygen saturation was $98 \%$ in room air. He had mild icteric sclera, and chronic venous stasis changes in bilateral lower extremities were noted. Bowel sounds were normal, and no hepatosplenomegaly or abdominal tenderness was noted on exam.

Laboratory investigations showed a hemoglobin count of $11 \mathrm{~g} / \mathrm{dl}$, white blood cell count of (WBC) $3800 \mathrm{cell} / \mathrm{mm}^{3}$, and platelet count of $214,000 / \mathrm{mm}^{3}$. Alanine aminotransferase (ALT) and aspartate aminotransferase (AST) were elevated at 326 and $321 \mathrm{IU} / \mathrm{L}$, respectively. Total bilirubin was $3.1 \mathrm{mg} / \mathrm{dl}$ with direct bilirubin of $1.8 \mathrm{mg} / \mathrm{dl}$. Alkaline phosphatase (ALP) was 1,219 IU/L with lipase $250 \mathrm{IU} / \mathrm{L}$. Renal function tests were at baseline at $1.72 \mathrm{mg} / \mathrm{dl}$ (baseline $1.7-1.9 \mathrm{mg} / \mathrm{dl})$. Recent upper and lower endoscopy (1 month earlier) did not show significant abnormalities, except for mild antral gastropathy. A computed tomography (CT) scan of abdomen and pelvis revealed stable pelvic adenopathy with largest lymph node measuring $4.4 \times 1.7 \mathrm{~cm}$ (which was noted on the earlier CT scan as well). The pancreatic tissue and abdominal vessels appeared normal. With concern for underlying malignancy, a lymph node biopsy was performed. Endoscopic retrograde of cholangiopancreatography revealed distal common bile duct stricture of $3 \mathrm{~cm}$ without obstruction, for which a biliary stent was placed. No pancreatic lesions were observed, and biliary brushings were negative for malignancy.

The patient returned to the ED two months later; this time with a fever $\left(102^{\circ} \mathrm{F}\right)$, nausea, and right upper quadrant pain for 2 days. Complete blood counts revealed elevated white cell count of $18,700 / \mathrm{mm}^{3}$. AST, ALT, and bilirubin levels were within normal limits, and ALP was elevated at $127 \mathrm{IU} / \mathrm{L}$. The CT scan of abdomen revealed intrahepatic biliary ductal dilatation and gall bladder wall thickening with pericholecystic inflammatory stranding with normal pancreas (Figure 1). He was started on broad-spectrum antibiotics with piperacillin-tazobactam. Urgent surgical decompression with percutaneous drain placement was performed for stent blockage (with re-stent placement) and bile duct stricture from unknown etiology.

Meanwhile, histopathology from the earlier right iliac lymph node biopsy revealed sinus histiocytic aggregates interlaced with lymphocytes and plasma cells (Figure 2(a)), majority of cellular constituents being plasma cells, characterized by oval cellular contours and eccentrically located nuclei (Figure 2(b)); this was confirmed by diffuse immunoreactivity for CD138 (Figure 2(c)). Chromogenic in situ hybridization for kappa light chain (Figure 3(a)) and lambda light chain (Figure 3(b)) showed a mixture of kappa and lambda-bearing cells (approximate kappa to lambda ratio of $3: 1$ ); this finding suggested that the plasma cells were reactive in nature, arguing against the possibility of a plasma cell neoplasm. In situ hybridization for EBV-encoded RNA (EBER) was interpreted as negative.

As there was no evidence to suggest a lymphoma or a plasma cell neoplasm, a diagnosis of IgG-related disease was considered. Additional immunohistochemistry showed

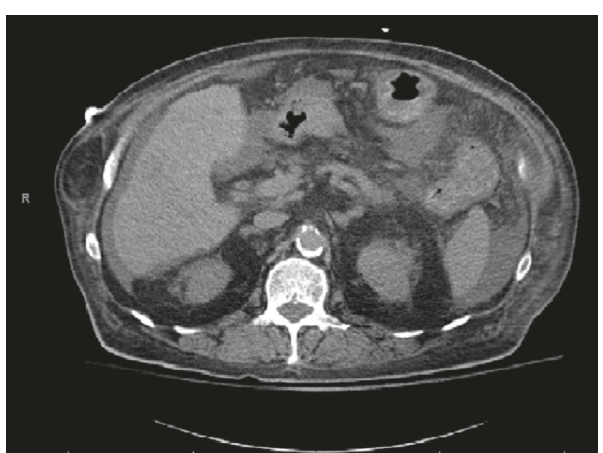

FIgURE 1: Gall bladder wall thickening with pericholecystic inflammatory stranding concerning for acute cholecystitis with normal pancreas.

that the plasma cells present expressed predominantly IgG (Figure 3(c)); of these, the majority (over 60\%) were reactive for IgG4 (Figure 3(d)). Serum protein electrophoresis and immunofixation showed polyclonal increase in IgG with no monoclonal proteins. Serum immunoglobulin (Ig) G levels were high $(3390 \mathrm{mg} / \mathrm{dl})$ with normal IgA and IgM. IgG subclasses were also high with IgG1 of 1800, IgG2 975, IgG3 324, and IgG4 $729 \mathrm{mg} / \mathrm{dl}$.

A diagnosis of IgG4-related sclerosing cholangitis was made, and he was started on prednisone $40 \mathrm{mg}$ daily for 6 weeks. A follow-up CT scan of abdomen after a month revealed significant reduction in the size of his pelvic lymph nodes with largest lymph node measuring $1.8 \times 0.7 \mathrm{~cm}$ (Figure 4). Endoscopic retrograde cholangiography (ERC) performed revealed resolution of stricture (Figure 5). The biliary stent was removed following resolution of the stricture. He remains on prednisone $20 \mathrm{mg}$ daily with close rheumatology follow-up. Rituximab therapy is being considered as a steroid-sparing agent.

\section{Description}

Although reported as a relatively rare disease with a prevalence of 100 per 100,000 adults based on limited studies done in Japan [7], the prevalence of IgG4-RD is likely higher due to underdiagnoses. Fortunately, increasing recognition among various specialties due to its multisystem involvement has been noted in recent years [8]. High index of suspicion for IGg4-RD should be raised in the setting of a constitution of clinical features such as pancreatitis (present in $60 \%$ of IgG4-RD) of unknown origin, sclerosing cholangitis (13\% of IgG4-RD), or bilateral salivary and/or lacrimal gland enlargement (34\% of IgG4-RD) [5, 9]. Proximal IgG4-SC (hilar and intrahepatic bile duct) may occur solely or in association with pancreatitis, whereas isolated lower duct IgG4-SC, as in our case, is rare and might pose a diagnostic challenge. It is more commonly observed with IgG4-RD affecting organs outside the pancreatobiliary system [5].

The differentiation of IgG4-SC from primary sclerosing cholangitis (PSC) and cholangiocarcinoma is clinically very important as IgG4-SC usually has a good response to steroid therapy [10]. IgG-SC patients are generally older than PSC 


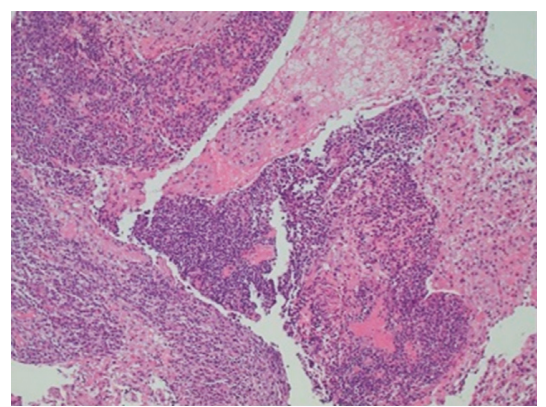

(a)

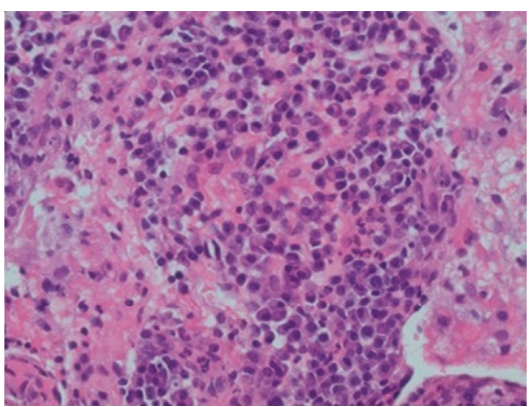

(b)

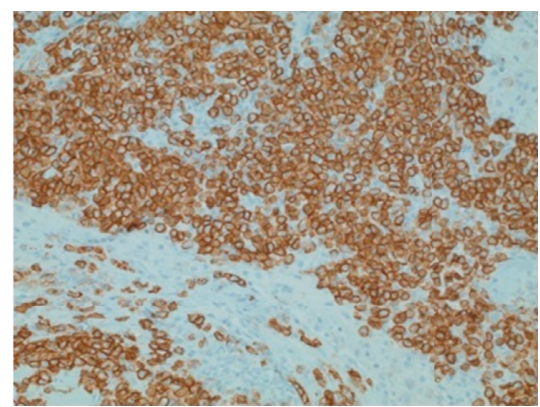

(c)

FIGURE 2: Biopsy fragment of right iliac lymph node showing sinus histiocytic aggregates interlaced with lymphocytes and plasma cells. (a) H\&E stain, 100x original magnification. Small cells within the lymph nodes, showing morphologic features of plasma cells, characterized by an oval cellular morphologic, and eccentrically placed nuclei. (b) H\&E stain, 200x original magnification. Majority of cellular constituents confirmed to be plasma cells, as demonstrated by diffuse reactivity for CD138 by immunohistochemistry. (c) 200x original magnification.

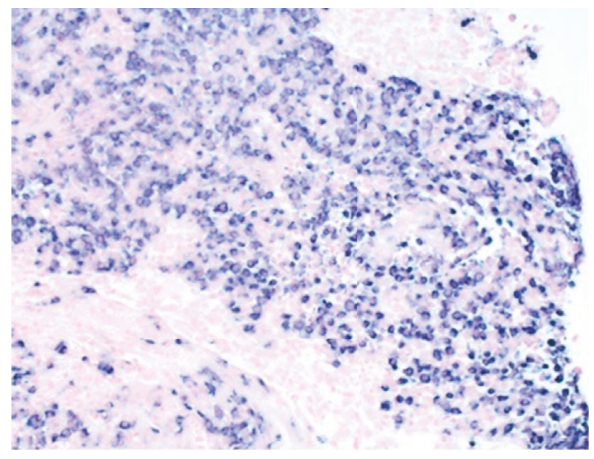

(a)

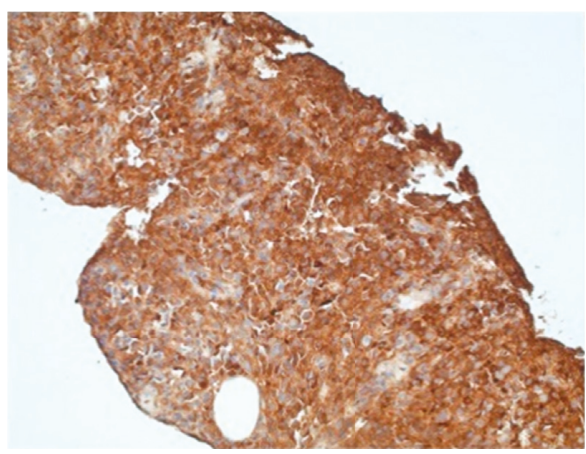

(c)

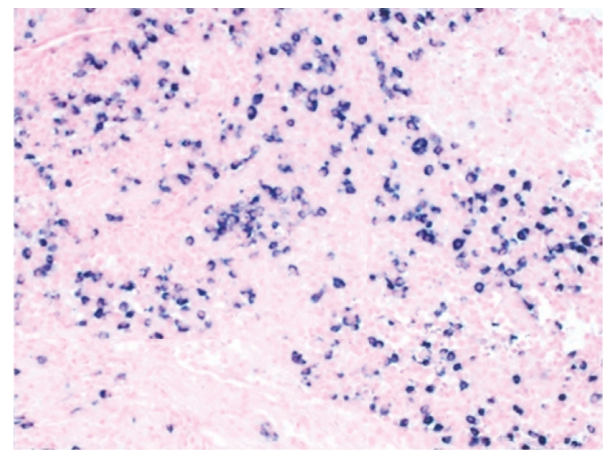

(b)

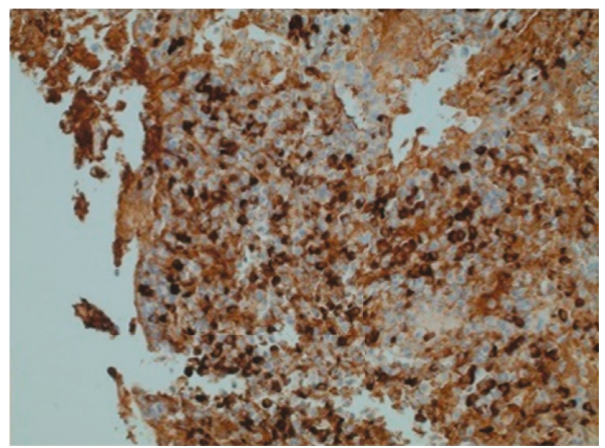

(d)

FIgURE 3: Chromogenic in situ hybridization showing a mixture of kappa-bearing plasma (a) and lambda-bearing plasma (b) cells, with an approximate kappa to lambda ratio of 3-4 to 1, appropriate for a physiologic/reactive phenomenon, without evidence of obvious restriction. Plasma cells expressed predominantly IgG (c); of these, the majority (over 60\%) were immunoreactive for IgG4 (d) (200x original magnification).

patients, with more than $90 \%$ being diagnosed at or above 60 years of age $[5,6,8,9]$. Also, unlike other autoimmune conditions, IgG4-SC is more common in males $(\mathrm{M}: \mathrm{F}=4: 1)$. Imaging features suggestive of PSC are multifocal intrahepatic duct involvement with beaded pattern secondary to short segmental strictures, whereas IgG4-SC has prominent wall thickening in the bile duct [10]. Similarly, imaging features of cholangiocarcinoma include solitary lesion with irregular margins with eccentric wall thickening and invisible bile duct lumen in the involved segment $[10,11]$.
The Japanese IgG4-RD committee has recently published a diagnostic criterion for IgG4-SC (2012) based on a combination of 4 features: (1) characteristic biliary imaging findings-diffuse swelling or organ mass, (2) elevation of serum IgG4 concentrations ( $\geq 135 \mathrm{mg} / \mathrm{dl})$, (3) coexistence of IgG4-related diseases, and (4) characteristic histopathological features of steroid therapy are optional criteria to confirm accurate diagnosis of IgG4-SC [8, 12]. Typical histopathologic features of IgG4-RD include marked lymphocytic and plasmacyte infiltration with high percentage of 


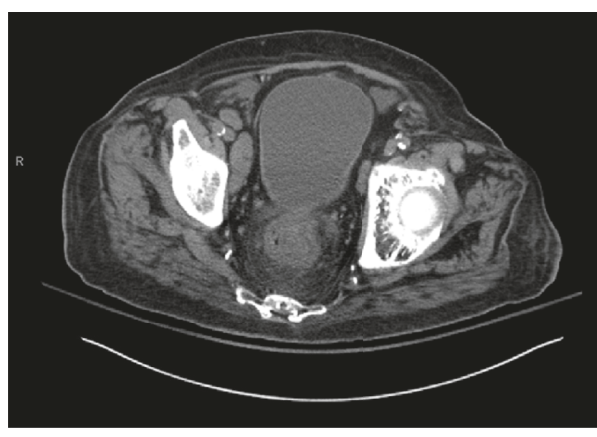

(a)

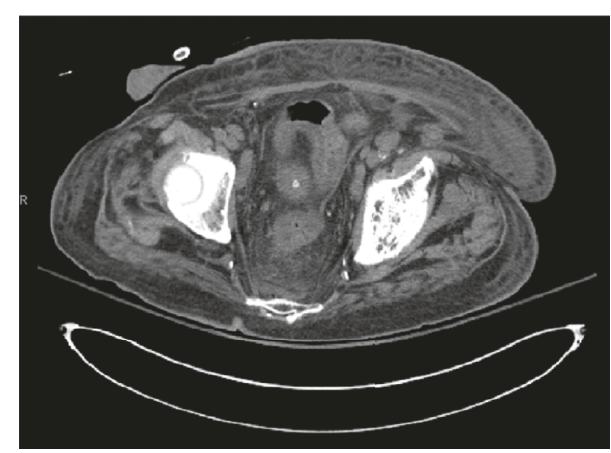

(b)

FIGURE 4: Top normal-sized pelvic lymph nodes decreased in size in repeat CT (b) compared to previous CT (a). The largest right external iliac lymph node measures $1.8 \times 0.7 \mathrm{~cm}$.

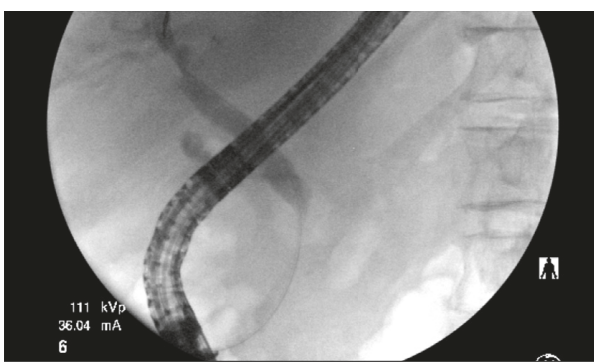

(a)

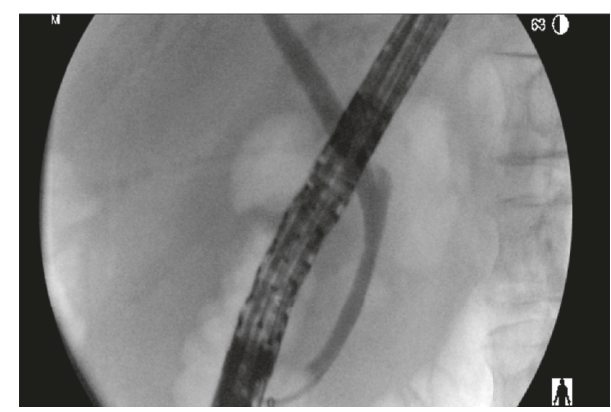

(b)

FIGURE 5: Endoscopic retrograde cholangiography showing presence of stricture in the biliary duct pre-ERC (a) compared to post-ERC (b).

IgG4-positive plasma cells, storiform fibrosis, and obliterative phlebitis [13]. In our case, the first two histopathologic features were observed.

Glucocorticoids remain the mainstay of therapy for remission induction in all patients with active IgG4-RD. Retrospective studies performed have shown good response to immunosuppression (prednisone at a dose of 30-40 mg per day), which generally leads to a rapid induction of disease remission $[2,5]$. In refractory cases or patients with difficulty in tapering prednisone (usually below $5 \mathrm{mg} /$ day), addition of steroid-sparing agents such as rituximab might be indicated. Although the natural history and long-term outcomes are not well understood, majority of patients tend to relapse, and most patients have a chronic disease course [14]. Hence, close follow-up with rheumatology is recommended.

\section{Conclusion}

Isolated IgG4-SC is a difficult entity to diagnose with vague presentation and overlapping symptoms with PSC, cholangiocarcinoma, other autoimmune conditions, and malignancy. Appropriate clinical, serologic, radiologic, and pathologic features may aid in diagnosis, although none in isolation is diagnostic. Glucocorticoids are the mainstay of therapy, and rituximab might be added as a steroid-sparing agent, especially in refractory or steroid-dependent cases.
Close follow-up is recommended due to its chronic and relapsing course.

\section{Conflicts of Interest}

The authors declare that they have no conflicts of interest.

\section{References}

[1] H. Hamano, S. Kawa, A. Horiuchi et al., "High serum IgG4 concentrations in patients with sclerosing pancreatitis," New England Journal of Medicine, vol. 344, no. 10, pp. 732-738, 2001.

[2] A. Tanaka, S. Tazuma, K. Okazaki et al., "Clinical features, response to treatment, and outcomes of IgG4-related sclerosing cholangitis," Clinical Gastroenterology and Hepatology, vol. 15, no. 6, pp. 920.e3-926.e3, 2017.

[3] T. Kamisawa, N. Funata, Y. Hayashi et al., "A new clinicopathological entity of IgG4-related autoimmune disease," Journal of Gastroenterology, vol. 38, no. 10, pp. 982-984, 2003.

[4] V. Thanthitaweewat, P. Chantranuwatana, and N. Chirakalwasan, "Immunoglobulin G4-related disease: a rare steroid-responsive disease," Respirology Case Reports, vol. 5, no. 3, article e00231, 2017.

[5] Y. Zen, Y. Nakanuma, and B. Portmann, "Immunoglobulin G4-related sclerosing cholangitis: pathologic features and histologic mimics," Seminars in Diagnostic Pathology, vol. 29, no. 4, pp. 205-211, 2012. 
[6] T. Nakazawa, Y. Ikeda, Y. Kawaguchi et al., "Isolated intrapancreatic IgG4-related sclerosing cholangitis," World Journal of Gastroenterology, vol. 21, no. 4, pp. 1334-1343, 2015.

[7] K. Uchida, A. Masamune, T. Shimosegawa, and K. Okazaki, "Prevalence of IgG4-related disease in Japan based on nationwide survey in 2009," International Journal of Rheumatology, vol. 2012, Article ID 358371, 5 pages, 2012.

[8] G. Beyer, T. Schwaiger, M. M. Lerch, and J. Mayerle, "IgG4related disease: a new kid on the block or an old aquaintance?," United European Gastroenterology Journal, vol. 2, no. 3, pp. 165-172, 2014.

[9] A. H. Nizar and E. Toubi, "IgG4-related disease: case report and literature review," Autoimmunity Highlights, vol. 6, no. 12, pp. 7-15, 2015.

[10] N. Seo, S. Y. Kim, S. S. Lee et al., "Sclerosing cholangitis: clinicopathologic features, imaging spectrum, and systemic approach to differential diagnosis," Korean Journal of Radiology, vol. 17, no. 1, pp. 25-38, 2016.

[11] J. H. Kim, J. H. Byun, S. J. Lee et al., "Differential diagnosis of sclerosing cholangitis with autoimmune pancreatitis and periductal infiltrating cancer in the common bile duct at dynamic CT, endoscopic retrograde cholangiography and MR cholangiography," European Radiology, vol. 22, no. 11, pp. 2502-2513, 2012.

[12] H. Ohara, K. Okazaki, H. Tsubouchi et al., "Clinical diagnostic criteria of IgG4-related sclerosing cholangitis 2012," Journal of Hepato-Biliary-Pancreatic Sciences, vol. 19, no. 5, pp. 536-542, 2012.

[13] K. E. G. O’Malley, S. B. Todd, C. Vladislav et al., "Histopathological findings in 29 lymph node biopsies with increased IgG4 plasma cells," Modern Pathology, vol. 25, no. 3, pp. 480-491, 2011.

[14] J. H. Stone, J. K. C. Chan, V. Deshpande, K. Okazaki, H. Umehara, and Y. Zen, "IgG4-related disease," International Journal of Rheumatology, vol. 2013, Article ID 532612, 2 pages, 2013. 


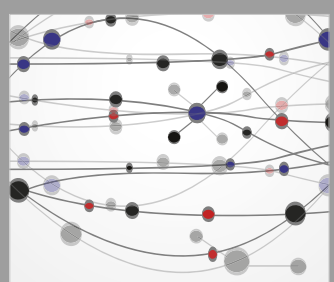

The Scientific World Journal
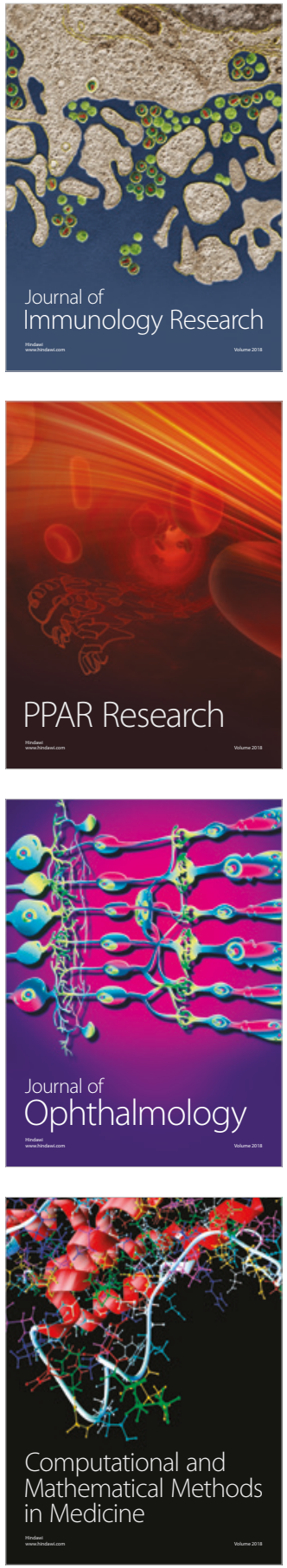

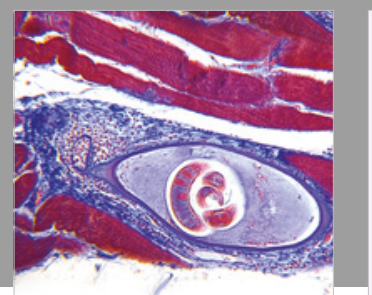

Gastroenterology Research and Practice

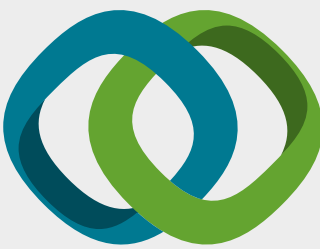

\section{Hindawi}

Submit your manuscripts at

www.hindawi.com
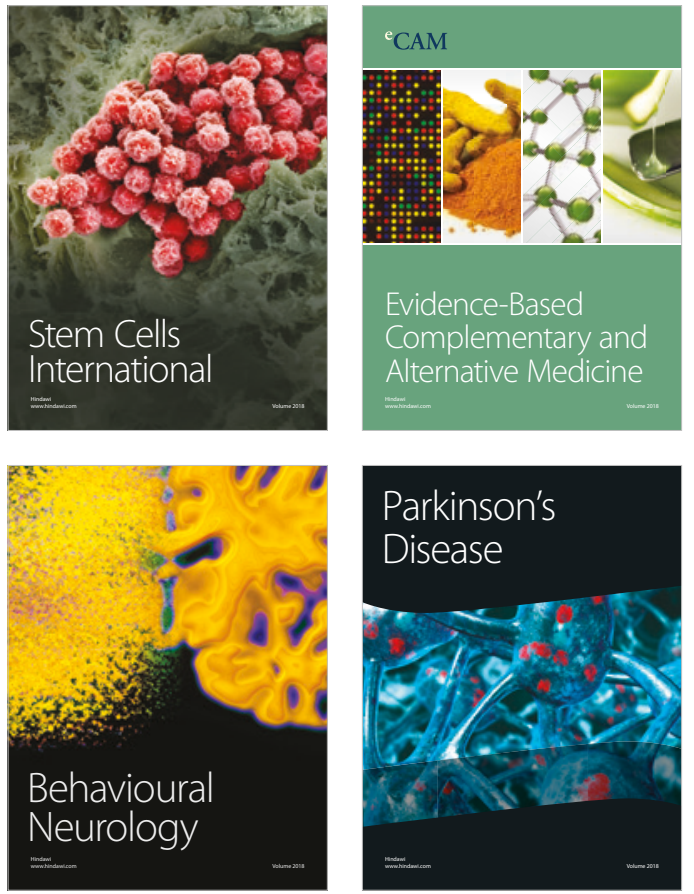

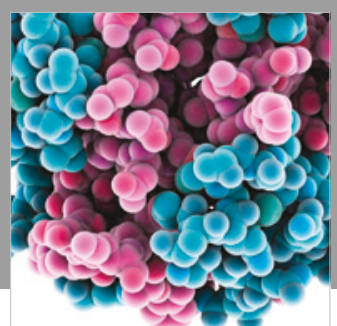

ournal of

Diabetes Research

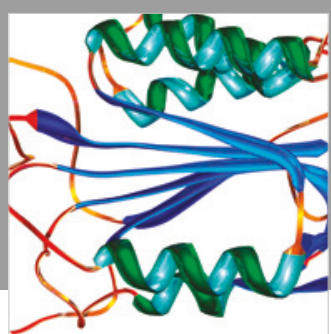

Disease Markers
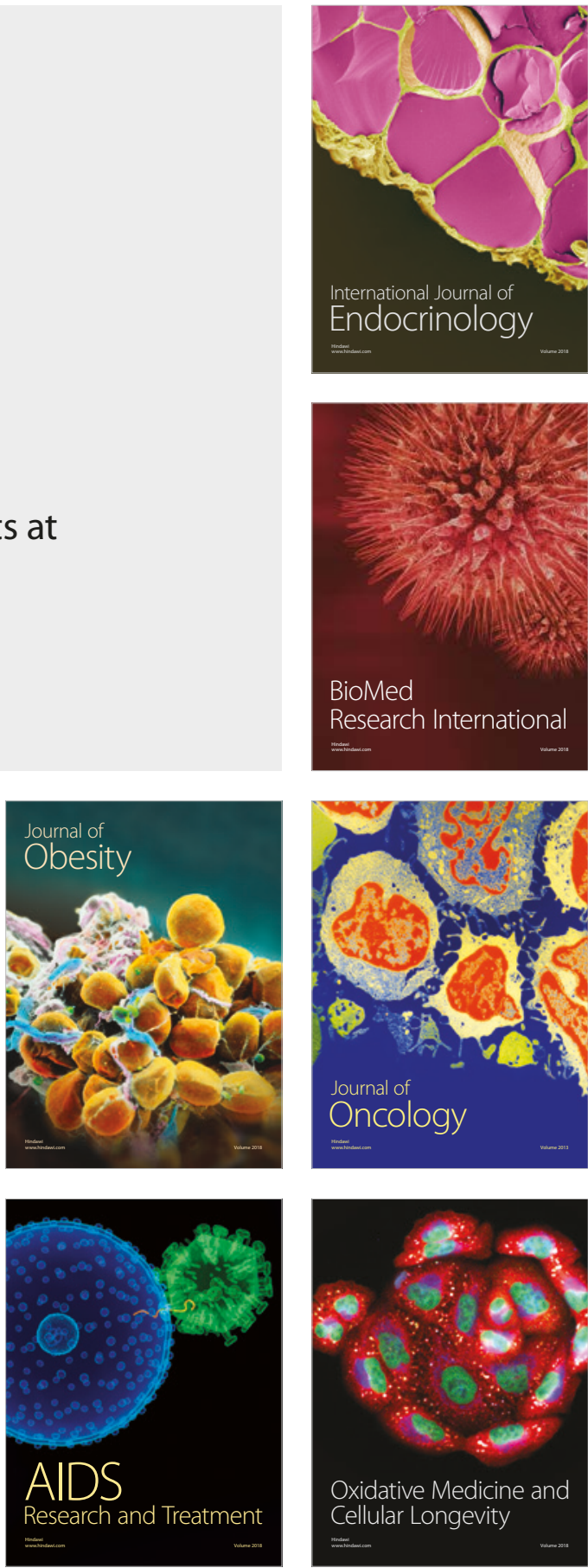